\title{
Late manifestation of laparoscopic injury of the ureter: a case presentation
}

\begin{abstract}
A case of late manifested injury of the left ureter after endometriosis surgery is presented. Thirty-six year old woman with history of two laparoscopies and a laparotomy all because of endometriosis was diagnosed left ovarian endometrioma. She underwent laparoscopic surgery for the fourth time. The left ovary was tightly adhered to the pelvic side wall peritoneum; endometrioma ruptured during examination. Cyst wall stripping and coagulation was performed. She had a febrile attack on the postoperative $17^{\text {th }}$ day. After a full check-up she was diagnosed as having urinary infection. Three days later she was presented again and was diagnosed left ureteral rupture. Because of the failure of ten days stenting, she underwent ureteroneocystostomy. Reviewing the pertinent literature and regarding our case, we concluded that, in order to avoid ureteral injury, high risk patients must be defined preoperatively and to save the patient from painstaking correction procedures all the possible measures must be taken.
\end{abstract}

Volume 2 Issue 3 - 2015

\author{
Hakan Kozinoğlu \\ Art Medical Center, Turkey
}

Correspondence: Hakan Kozinoğlu, Art Medical Center, Istanbul,Turkey, Tel 90532257 9355, +90 212219 I202, Email hkozinoglu@gmail.com

Received: March 16,2015 | Published: April 15, 2015

Keywords: laparoscopy, endometriosis, ureter injury

Abbreviations: IVP, intravenous pyelography; LAVH, laparoscopically assisted vaginal hysterectomy

\section{Introduction}

Vascular or intestinal injuries in laparoscopy generally occur during the entry of the trocars and majority of the mare noticed intraoperatively and are managed appropriately. But urinary system injuries, especially pertinent to ureters is mostly related to the procedure performed and majority of them manifest postoperatively. The first reported complication of ureteral injury in laparoscopic surgery was presented by Stengel et al. ${ }^{1}$ Urinary tract injuries have been reported to occur $0.25 \%$ in major laparoscopic operations. ${ }^{2}$

A case of late manifested injury of the left ureter after endometriosis surgery is presented here. Reviewing the pertinent literature as well, lessons are taken and some recommendations are stated.

\section{Case report}

Thirty-six years old primary infertile woman was presented with lower abdominal pain more prominent on her leftside. Her medical history was eventful with a laparoscopic right ovarian endometrioma removal in the year 2000, laparotomy and partial resection of the right ovary in the year 2002 and another laparoscopy in the year 2004 for the left ovarian endometrioma removal. During that 4 years of course, she had two in vitro fertilization cycles, both were failed.

Transvaginal scanning revealed an endometrioma of $7 \mathrm{~cm}$ in the left ovary. Transabdominal sonographic scanning of intra and retroperitoneal organs (kidneys) was done in the radiology department and was reported as normal. CA-125 level was $277 \mathrm{U} / \mathrm{mL}$, routine biochemistry was normal. She was offered a laparoscopy for the third time.

During the laparoscopy, the abdomen was unexpectedly almost adhesion free. Only the left ovary was tightly adhered to pelvic sidewall due to the endometrioma beneath. Ovary was examined using endoscopic palpator, during with endometrioma was drained. The endometriotic cystwall stripped and coagulated with bipolar cauterization. Then, the peritoneum on the adhesion site was coagulated with one shot of bipolar cautery. Following the haemostasis, abdomen was irrigated several times. The postoperative 2 days was uneventful and the patient discharged from the hospital. On the postoperative $17^{\text {th }}$ day, she presented with a febrile attack of $39^{\circ} \mathrm{C}$ and lower abdominal pain. Diagnostic work uprevealed leucocytosis and pyuria and slight hematuria. The patient was consulted with urology department. Renal sonogram was normal, intravenous pyelography (IVP) showed normal ureteral flow on both sides. The patient was diagnosed as urinary infection and third generation cephalosporin treatment was started. In 12 hours, fever resolved and the symptoms disappeared. She was discharged from the hospital on her demand and the antibiotic treatment continued orally.

On the $21^{\text {st }}$ postoperativeday, she was presented again with fever and the symptoms of peritonitis and bilateral flank pain. Transvaginal sonography revealed fluid in the Douglas pouche. A culdocentesis was performed and the fluid was reported containing high urea $(107 \mathrm{mg} / \mathrm{dL})$ and high creatinin $(6.4 \mathrm{mg} / \mathrm{dL})$. A new IVP showed extravasation of contrast dye in the lowerpart of the left ureter (Figure 1). She transferred to urology department and underwent cystoscopic double-J stenting. Ten days of stenting did not lead to healing. On the $31^{\text {st }}$ postoperative day, she underwent ureteroneocystostomy operation. Ureteral catheter was removed at the sixth postoperative week. Imaging at the third postoperative month was normal.

After all, the video laparoscopic recording of the patient was reviewed by department board consisted of three professors to clarify whether the excessive liberation procedure or the thermal injury caused by coagulation led to the rupture of the ureter. None of them were obviously responsible. Either of them might have caused the weakening of the ureteral wall eventually leading to necrosis. 


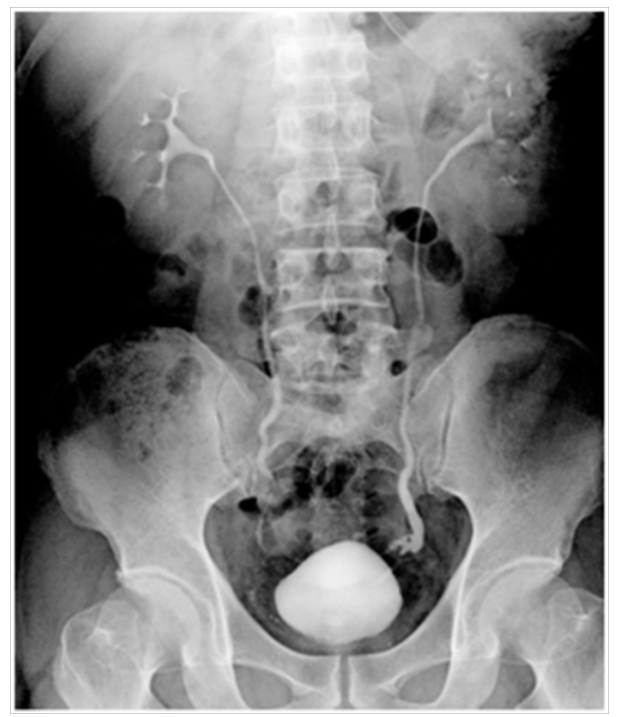

Figure I Pyelogram showing the leakage in the left ureter distalend.

\section{Discussion}

Late presentation of 12 ureteral injury cases was reported by Oh et al. ${ }^{3}$ Manifestations of the cases were between 3 and 33 days postoperatively. They advocated early open and repair instead of ureteral stenting. In the current case initial double-J stenting failed accordingly. Tamussino et al. ${ }^{4}$ reported 4 ureteral complications, all was during laparoscopically assisted hysterectomies. ${ }^{4}$ Of those two was presented in late postoperative period. They pointed out the risk of increased ureteral injury when dissection performed below the uterine vessels. Terzibachian et al. ${ }^{5}$ recommended intraoperative stenting of the high risk patients.

The author of the current case presentation has performed 3917 operative laparoscopy between the years 1994 and 2012. Of those, 1841 were major operative laparoscopy including laparoscopically assisted vaginal hysterectomy (LAVH), adnexial mass removal, adhesiolysis in stage III or IV endometriosis, subserousal myomectomy and extrauterine pregnancy. The presented case is the only ureteral injury in eighteen years. The most prominent risk factor increasing ureteral injury during laparoscopy reported to be endometriosis. ${ }^{6}$

Ostrzenski et al. ${ }^{7}$ published a review on laparoscopic ureteral injury in pelvic surgery. ${ }^{7}$ In their series of 70 cases, the incidence of injury was reported as 1-2 \%, majority was presented postoperatively $(70 \%)$. The leading cause was laparoscopically assisted vaginal hysterectomy $(20 \%)$.

Regarding the literature and current case we concluded that, in order to avoid ureteral injury, high risk patients must be defined preoperatively. These are benign surgery like endometriosis, repeat surgery, pelvic inflamatory disease and all procedures performed around distal ureter.

In order to save the patient from pains taking morbidity of late ureteral complications, the best approach will be opening the broad ligament first and freeing the ureter from sidewall peritoneum. Then, affected peritoneum can be resected or coagulated. If not experienced, taking following measures can be recommended: preoperatively give patient a dye to color the urine and to trace the ureters, ask for intraoperative urologic consultation for possible intraoperative catheterization of the ureters.

\section{Acknowledgments}

None.

\section{Conflicts of interest}

The authors declare there is no conflict of interests.

\section{References}

1. Stengel JN, Felderman ES, Zamora D. Ureteral injury: complication of laparoscopic sterilization. Urology. 1974;4(3):341-342.

2. Horkki-Siren P, Sjoberg J, Kurki T. Major complications of laparoscopy: a follow-up Finnish Study. Obstet Gynecol. 1999;94(1):94-98.

3. Oh BR, Kwon DD, Park KS, et al. Late presentation of ureteral injury after laparoscopic surgery. Obstet Gynecol. 2000;95(3):337-339.

4. Tamussino KF, Lang PF, Breinl E. Ureteral complications with operative gynecologic laparoscopy. Am J Obstet Gynecol. 1998;178(5):967-970.

5. Terzibachian JJ, Gay C, Bertrand V, et al. Value of ureteral catheterization in laparoscopy. Gynecol Obstet Fertil. 2001;29(6):427-432.

6. Saidi MH, Sadler RK, Vancaillie TG, et al. Diagnosis and management of serious urinary complications after major operative laparoscopy. Obstet Gynecol. 1996;87(2):272-276.

7. Ostrzenski A, Radolinski B, Ostrzenska KM. A review of laparoscopic ureteral injury in pelvic surgery. Obstet Gynecol Surv. 2003;58(12):794799. 